\title{
Hermann MÜller (Thurgau) - Würzburgs heimlicher Star
}

\author{
FRANZISKA WIEGAND
}

\begin{abstract}
HERMANN MÜLler was born in Thurgau (Switzerland) and became a plant physiologist, oenologist and plant breeder. He was $\mathrm{PhD}$ student of the prominent plant physiologist JULIUS VON SACHS at the University of Wuerzburg. His name became famous through the widespread grape cultivar 'Müller-Thurgau'. Nowadays, people tend to associate the name 'Müller-Thurgau' only with the white wine rather than thinking of the person behind it.
\end{abstract}

\section{Zusammenfassung}

Hermann Müller, der 1850 im Thurgau in der Schweiz geboren wurde, war Pflanzenphysiologe, Önologe und Rebzüchter. Als Schüler des bekannten Pflanzenphysiologen Julius vON SACHS promovierte, forschte und lehrte er an der Universität in Würzburg. Einige Jahre später züchtete er mit dem 'Müller-Thurgau' eine der bekanntesten Reb-Sorten für Weißwein und machte Würzburg damit ein großes Geschenk. Allgemein bekannt ist heute noch der Wein, nicht aber der Mensch MüLLERTHURGAU.

\section{MÜller-ThurgaU - sein Leben}

HERMANN MÜLLER erblickte als Sohn eines Bäkkermeisters und Winzers am 21.10.1850 in Tägerswilen (Kanton Thurgau, Schweiz) das Licht der Welt. Bereits mit 19 Jahren war er als Lehrer an der Realschule in Stein am Rhein tätig. Diese Arbeit befriedigte MÜLLER jedoch nicht gänzlich, so dass er nach zwei Jahren am Polytechnikum Zürich (heute ETH) zu studieren begann. 1872 schloss er sein Diplom als Fachlehrer für Naturwissenschaften ab, lehrte kurzzeitig in Kreuzlingen und ging anschließend zum Studium der Botanik nach Würzburg. Nun begann MüLlERs wissenschaftliche Laufbahn. Er wurde bei dem berühmten Botaniker JULIUS VON SACHS mit dem Thema „Die Sporenvorkeime und Zweigvorkeime bei Laubmoosen" promoviert. Mit gerade einmal 24 Jahren war MÜLLER nun Doktor der Philosophie. Zur damaligen Zeit gehörten die Naturwissenschaften zu den philosophischen Instituten. Nach weiteren zwei Jahren als wissenschaftlicher Assistent von SACHS, in der er die Kälteresistenz und -empfindlichkeit von Pflanzen untersuchte, erhielt MÜLLER einen Ruf nach Geisenheim am Rhein. Dort leitete er die pflanzenphysiologische Abteilung der Königlichen Lehranstalt für Obst- und Weinbau und wurde später zum Professor ernannt. MÜLLER heiratete 1881 Berta Biegen, die Tochter eines Wein-

Abb. 1: Hermann Müller (Thurgau). händlers. Drei Töchter gingen aus dieser Verbindung hervor. Das Jahr 1890 war für MÜLLER ein wichtiges Jahr, denn er wurde Ehrenmitglied des Deutschen Weinbauverbandes und entschloss sich, zurück in seine Heimat nach Wädenswil zu gehen, um eine Weinbauanstalt aufzubauen. Erst

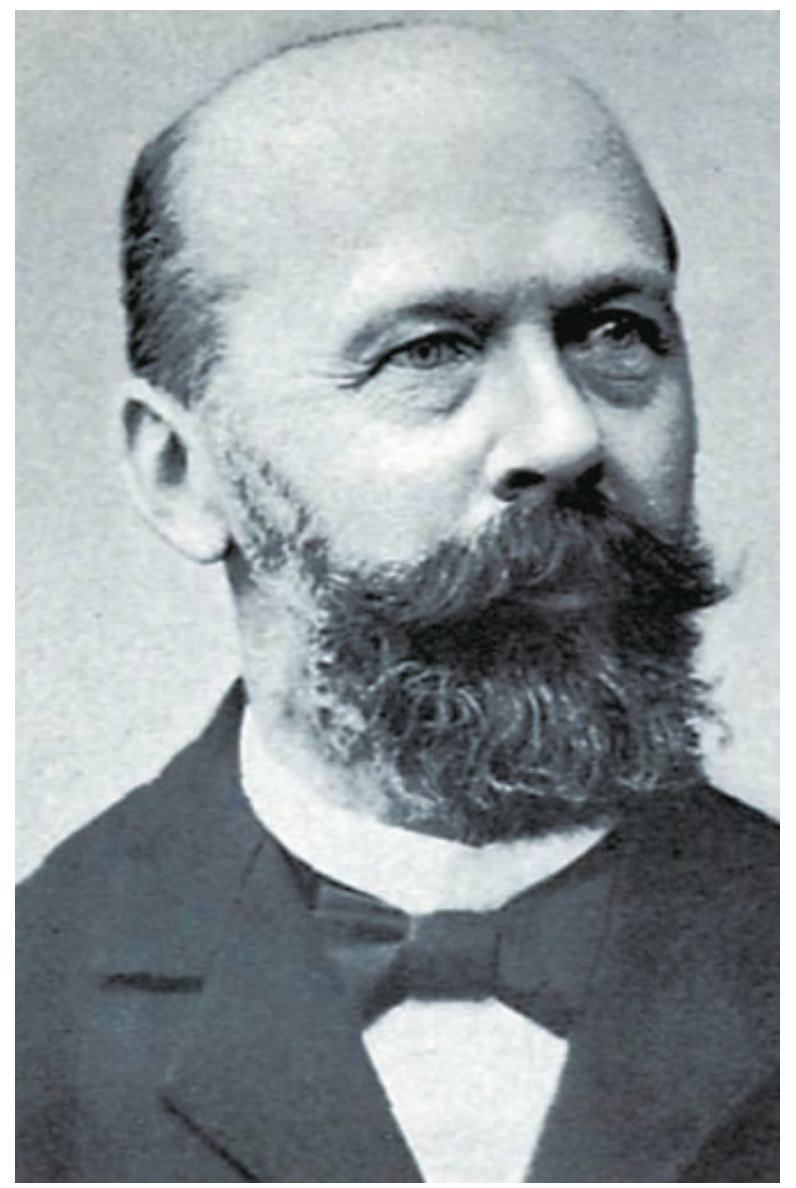




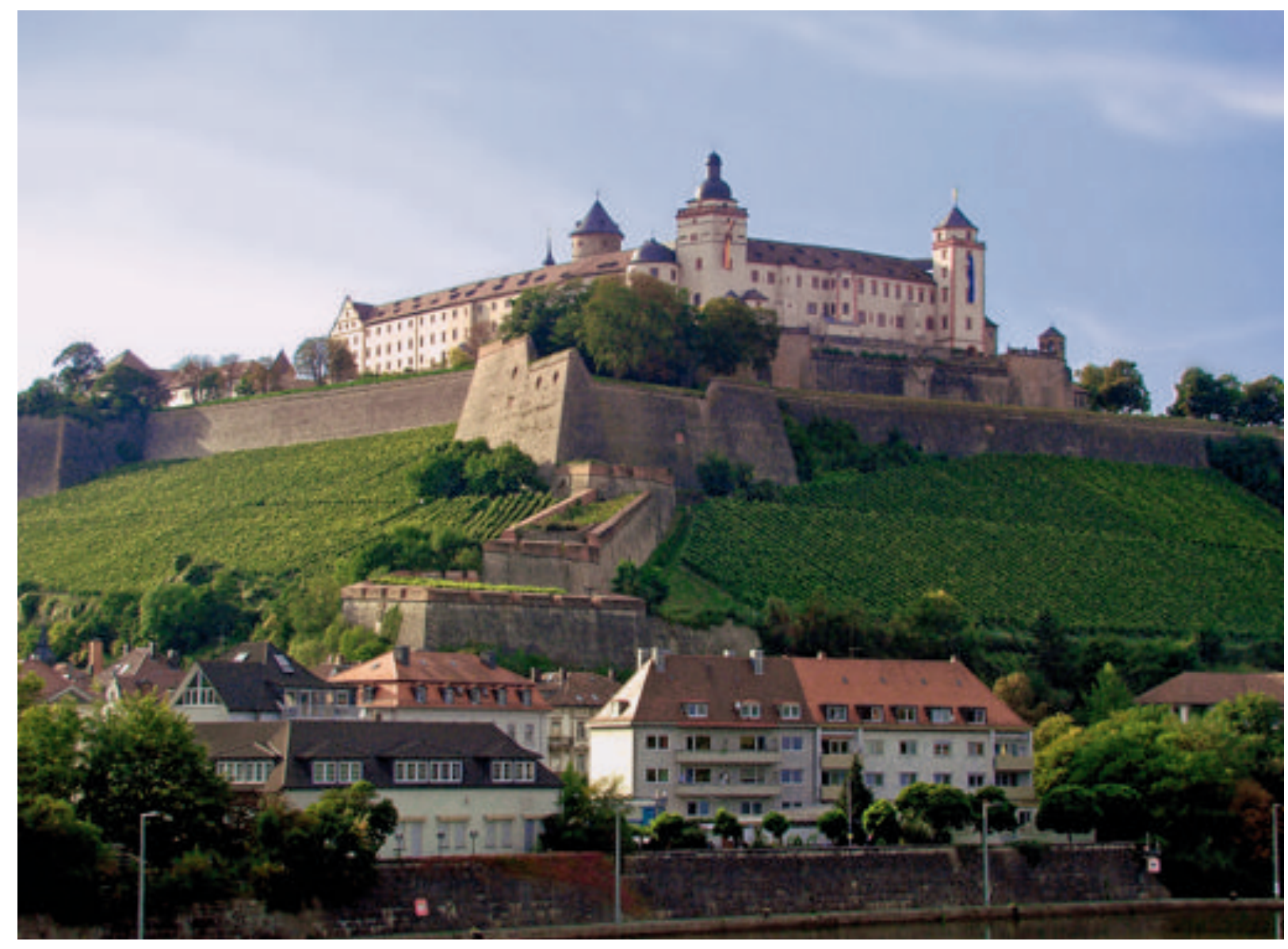

im Alter von 73 Jahren ging MÜLLER, welcher sich selbst MÜlLER-ThURGAU nannte, in den Ruhestand und starb am 18. Januar 1927. Sein Vermächtnis für die Allgemeinheit war eine bekannte Weinsorte. Für Botaniker und Weinbauern schrieb MÜller-ThurgaU insgesamt 500 Veröffentlichungen.

\section{MÜller-ThurgaU - der Pflanzenphysiologe}

Wie viele andere Wissenschaftler zu jener Zeit wollte Hermann Müller-Thurgau an die Universität Würzburg. Hier lehrte und forschte am Botanischen Institut der bekannte, aber strenge Pflanzenphysiologe Julius VON SACHS. Dieser suchte sich seine Schüler und Doktoranden sehr genau aus. MÜLLER-THURGAU brachte schon naturwissenschaftliche Grundlagen mit, welche sich seine Kollegen noch erarbeiten mussten. Wichtige Eigenschaften für die Arbeit als Pflanzenphysiologe waren, damals wie heute, eine gute Beobachtungsgabe, chemische und physikalische Kenntnisse und Ehrgeiz. MÜLler-ThurgaUs Ar- beit wurde mit "summa cum laude", also mit höchstem Lob, bewertet.

Im Rahmen seiner Dissertation befasste er sich mit der Keimung von Laubmoosen. Als Wissenschaftlicher Assistent widmete er seine Zeit der Erforschung der Frostresistenz von WeinrebenKnospen. Der Einfluss seines Mentors brachte ihn hin zum Weinbau. Spätere Publikationen über die mineralische Ernährung (1879), den Stofftransport innerhalb der Pflanze (1879), die Zuckerbildung in der Weinbeere (1887) und Arbeiten zu Krankheiten des Weines (1888 und 1901) verdeutlichen die Prägung des Schülers. Auch SACHS befasste sich mit Krankheiten des Weines (1855 und 1874), mit Nährstoffen in Pflanzen (1861), sowie mit der Entstehung von Stärke in Blättern (1863). Eines konnte SACHS seinem „kleinen Schweizer”, wie er ihn nannte, jedoch nicht beibringen, nämlich das Zeichnen. SACHS war Sohn eines Kupferstechers und ein

Abb. 2: Anbau des 'Müller-Thurgau'-Weines in Würzburg. 
hervorragender Zeichner. Als Botaniker skizzierte man die Bilder für Veröffentlichungen damals eigenhändig. Müller-Thurgau war in dieser Hinsicht weniger talentiert und ließ zeichnen.

Die bereits zum damaligen Zeitpunkt florierende Weinregion Unterfranken mit der Hauptstadt Würzburg trug sicherlich auch zu MülLER-Thurgaus Forschungsvorlieben bei. Die Weinrebe und deren Popularität machten sie zu einem dankbaren Objekt der Forschung. In Geisenheim untersuchte er nicht nur die Morphologie der Weinreben und deren Krankheiten, sondern auch Gärungsprozesse durch Hefen. Die Gärungsphysiologie ist für den Weinbau und die -produktion unabdingbar. Müller-ThurgaU war mit seinen ca. 111 Studien (u. a. „Die Herstellung unvergorener alkoholfreier Obst- und Traubenweine", 1896) dazu sehr erfolgreich. SACHS dagegen hatte sich nie mit Hefen auseinander gesetzt.

\section{3. 'Müller-Thurgau' - der Wein}

"Wie wichtig könnte zum Beispiel für manche Weinbaugegenden eine Traubensorte werden, die mit den köstlichen Eigenschaften der Rieslingtraube die sichere und frühe Reifezeit des Sylvaners vereinigte!" (MÜLLER-THURGAU, 1883)

Zunächst war es nur eine Idee von MÜLlERThurgau. Doch noch im selben Jahr machte er sich mit seinem Assistenten SCHELLENBERGER an die Umsetzung. Sie kreuzten einen Weißen Riesling (Mutter) mit einem Grünen Silvaner (Vater). Die Kreuzung erbrachte 150 Produkte, doch vorerst fand die Hybride keine Beachtung. Als Müller-Thurgau 1890 zurück in die Schweiz nach Wädenswil ging, nahm er seine KreuzungsProdukte mit. Dort angekommen, propagierte er sein Werk. Er wollte nicht, dass der Wein in der Schweiz nach ihm benannt wurde. Der Wein wurde demzufolge nach seinen Eltern als Rivaner oder Riesling x Silvaner bezeichnet. Erst 23 Jahre später wurde die Rebsorte von einem Inspektor für Weinbau namens AUGUST DERN nach Franken (Sendelbach bei Lohr) gebracht und angebaut. Im selben Jahr würdigte man HERMANN MÜLlER, indem die Rebsorte nun offiziell als 'Müller-Thurgau' benannt wurde. 1925 wurde die Rebe dann am Schlossberg der Mari- enburg in Würzburg gepflanzt, eine Sternstunde für den Weinbau der Stadt. Heute noch handelt es sich um die älteste Anpflanzung der Rebsorte 'Müller-Thurgau' in Franken von 1925. Nun begann der Siegeszug des Weines in Deutschland.

In den 1970er Jahren hatte der Anbau von 'Müller-Thurgau' seinen Höhepunkt erreicht. Mehr als die Hälfte der Anbaufläche Frankens war zwischenzeitlich von ihm dominiert. Heute sind es $30 \%$ der Fläche. 'Müller-Thurgau' ist damit noch vor 'Silvaner' (20\%) und 'Bacchus' (12\%) die Nummer 1. Zwar hat der Wein eine milde Säure und ist fruchtig, allerdings sind ihm 'Silvaner' und 'Bacchus' als leichte Sommerweine dicht auf den Fersen. Aufgrund seiner Frostresistenz (von Bedeutung in den letzten besonders kalten Wintern) und des hohen Ertrags gegenüber seinen Konkurrenten kann man jedoch annehmen, dass der 'Müller-Thurgau' eine Renaissance erlebt.

In Deutschland wird 'Müller-Thurgau' mit einer Fläche von 4300 ha hauptsächlich in Rheinhessen angebaut, die Anbauflächen in Franken hingegen umfassen 1800 ha. 'MüllerThurgau' ist nach dem Riesling der am zweithäufigsten kultivierte Wein.

Europaweit befinden sich mit insgesamt 8000 ha die größten 'Müller-Thurgau'-Flächen in Ungarn. In Neuseeland, den USA und sogar in Japan ist der 'Müller-Thurgau' angekommen. Mit diesem weltweiten Erfolg leidet auch das Image der Sorte. Es werden immer mehr minderwertige Produkte auf den Markt gebracht und somit entseht für den Konsumenten der Eindruck, es handle sich um einen Massenwein.

\section{4. 'Müller-Thurgau' und die Elternfrage}

Bis ins Jahr 2000 beschäftigte sich die Wissenschaft mit der Frage nach den wahren Eltern des 'Müller-Thurgau'. Fachleute zweifelten aufgrund morphologischer Merkmale an der angegebenen Elternschaft der Rebe. 1994 bewiesen BÜSCHER et al. (1994) mittels molekularbiologischer Methoden die Mutterschaft des Rieslings, schlossen eine Vaterschaft des Silvaners jedoch klar aus. Langsam kam man dem Rätsel auf die Spur. Ein „,adeliger“" Vorfahr aus dem Kreise der Gutedel-Weine wurde als Vater herauskristallisiert. 


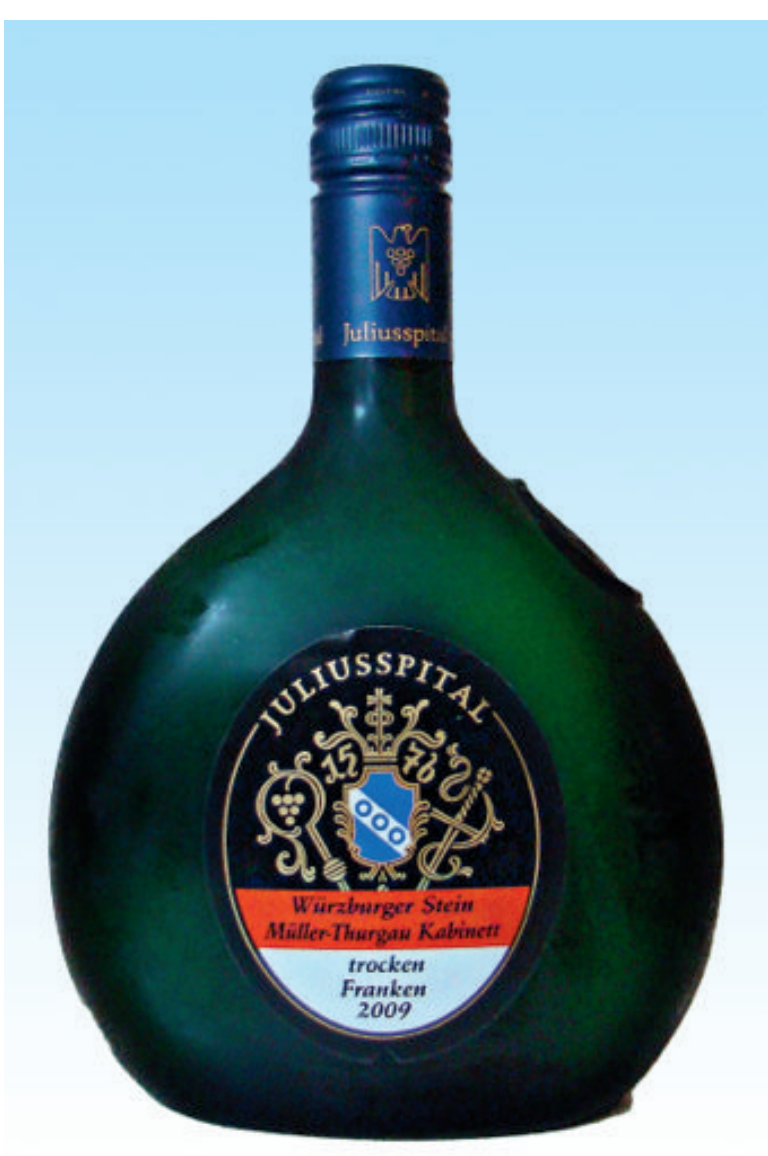

Genau 150 Jahre nach der Geburt des Schöpfers Hermann MÜller brachte die Analyse (MikroSatelliten-DNS) der Chromosomen aus BlattZellen der Rebe einen Vater mit dem weiblichen Namen 'Madelaine Royale' (Königliche Magdalenentraube) ans Licht (DETTWEILER et al. 2000).

\section{5. 'Müller-Thurgau' - Würzburgs Star}

Am 21. Oktober 2000 wurden zum 150. Geburtstag Hermann Müllers Person und seine Arbeit in Würzburg gefeiert und gewürdigt. So taten sich die Traditionsweingüter "Juliusspital", „Bürgerspital zum Heiligen Geist" und die"Staatliche Hofkellerei“ zusammen und produzierten einen gemeinsamen 'Müller-Thurgau-Bocksbeutel' mit dem Namen "Die Drei”. Diese kurzfristige Fusion diente nicht ausschließlich der Traditionspflege. Sie sollte auch das Image des Weines und des Anbaugebietes Mainfranken aufbauen. Das Jubiläum war auch später Anlass, eine Gedenktafel für Prof. Dr. Hermann MüLLER-THURGAU an seiner Würzburger Wirkungsstätte in der Klinikgasse anzubringen.
Trotz allem, fragt man die Menschen auf Würzburgs Straßen, so kennen sie den Wein 'Müller-Thurgau'. Dass sich dahinter aber auch ein sehr begabter Wissenschaftler verbirgt, ist ihnen fremd. Durch die weltweite Beliebtheit der Weinsorte sind Würzburg und Mainfranken eine der bekanntesten Anbaugebiete für frische, fruchtige Weißweine Deutschlands, und das trotz mancher Krisen und Globalisierung. Ohne HERMANN MÜLLERs brilliante Ideen wäre Würzburg als Weinstadt mit seinen drei großen Weingütern nicht das, was sie heute ist.

\section{Literatur}

BAUMgarT, P. 1995: Lebensbilder bedeutender Würzburger Professoren. 8, 129-156. - Neustadt a.d. Aisch.

BÜscher, N., Zyprian, E., Bachmann, O. \& Blalch, R. 1994: On the origin of the grapevine variety Müller-

Thurgau as investigated by the inheritance of random amplified polymorphic DNA (RAPD). - Vitis 33: 15-17. DetTweiler, E., Jung, A., Zyprian, E. \& Töpfer, R. 2000: Grapevine cultivar Müller-Thurgau and its true to type descent. - Vitis 39: 63-65.

Deutsches Weininstitut 2009: Deutscher Wein. Statistik 2009/2010: 6-7. - Mainz.

Fritsche, R., Heberlein, F. \& Schmid, H. 1974: Hermann Müller-Thurgau (1850-1927) und weitere Pioniere der Qualitätsverbesserung des Weines und der unvergorenen Trauben- und Obstsäfte. - Verein für Wirtschaftshistorische Studien. Schweizer Pioniere der Wirtschaft und Technik 29: 9-64.

Gimmler, H. 2001: Vom Moos zum Wein, vom Main zum Rhein: Würzburg und “sein” MÜller-Thurgau. Sonderheft zum 150. Geburtstag Dr. Hermann Müller-ThurGAUs. - Würzburg.

MÜller, H. (Thurgau) 1874: Die Sporenvorkeime und Zweigvorkeime der Laubmoose. - Dissertation Universität Würzburg. - Arb. Bot. Inst. 1: 475-599.

Müller-Thurgau, H. 1883: Der Weinbau 9. -

Würzburg.

SChaller, K. 2007: Die Tätigkeit von Prof. Dr. Dr. h.c. Hermann Müller-Thurgau. - Würzburg.

Schwab, A. o. J.: Prof. Dr. Dr. h. c. Hermann Müller (THURGAU). Lebenslauf. - Veitshöchheim. Statistisches Bundesamt 2008: Beschreibende Sortenliste des Bundessortenamtes. - Wiesbaden.

Abb. 3: Fränkischer Bocksbeutel mit 'Müller-Thurgau'Kabinett-Wein. 